\title{
Effects of laminar shear stress versus resveratrol on the citrulline-NO cycle in endothelial cells
}

\author{
Sue Im Jang, Yong Chool Boo* \\ Department of Molecular Medicine, Cell and Matrix Research Institute, BK21 Medical Education Program for Human Resources, \\ School of Medicine, Kyungpook National University, Daegu, South Korea \\ Email: ${ }^{*}$ cbooo@knu.ac.kr
}

Received 16 October 2012; revised 17 November 2012; accepted 26 November 2012

\section{ABSTRACT}

Laminar shear stress (LSS) due to pulsatile blood flow enhances endothelial function by multiple mechanisms including NO production. Red wine and its constituent, resveratrol, have also been postulated to provide vascular protective effects. The aim of the present study was to compare the effects of mechanical LSS and pharmacological resveratrol treatments on the endothelial citrulline-NO cycle. Human umbilical vein endothelial cells (HUVECs) were treated

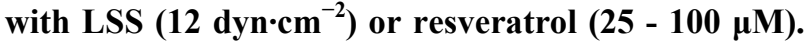
The expressions of argininosuccinate synthetase 1 (ASS1), argininosuccinate lyase (ASL), nitric oxide synthase 3 (NOS3) and cationic amino acid transporter 1 (CAT1), and the production of NO were determined. The expressions of Kruppel-like factor (KLF) 2 and KLF4 as upstream regulators of ASS1 and NOS3 were also analyzed. LSS strongly increased the mRNA levels of ASS1 (8.3 fold) and NOS3 (5.4 fold) without significant effects on ASL and CAT1 mRNAs. Resveratrol increased the ASS1 mRNA level in a dose-dependent manner up to 3.8 fold at $100 \mu \mathrm{M}$. The effects of resveratrol on the expressions of KLF2 and KLF4 mRNAs were smaller than those of LSS. Protein levels of ASS1 and NOS3, and NO production were markedly increased by LSS but resveratrol (50 $\boldsymbol{\mu M}$ ) increased only ASS1 protein level. The results of the current study showed that LSS had greater effects on the citrulline-NO cycle activity leading to NO production, compared to resveratrol. Because resveratrol was not so effective at stimulating the endothelial citrulline-NO cycle, further studies are needed to find more potent drugs that increase the expression of ASS1 and NOS3 genes.

Keywords: Citrulline-Nitric Oxide Cycle; Endothelial Cells; Laminar Shear Stress; Resveratrol

\footnotetext{
${ }^{*}$ Corresponding author.
}

\section{INTRODUCTION}

The endothelium, a monolayer of endothelial cells covering the internal surface of blood vessels, cardiac valves, and numerous body cavities, provides a semi-permeable barrier between circulating blood and the rest of the vessel wall [1]. It plays a key role in vascular physiology through production of a variety of substances involved in vasodilatation, blood coagulation, inflammation and immune response [2]. Therefore, the structural and functional integrities of the endothelium are essential for vascular health.

Nitric oxide (NO) produced by nitric oxide synthase 3 (NOS3) in the endothelium plays a key role in vascular physiology. NO regulates vascular tone as "endotheliumderived relaxing factor" [3] and inhibits the atherogenic events such as endothelial cell injury and activation, adhesion and transmigration of monocytes through the inflamed endothelium, and proliferation of underlying smooth muscle cells [4].

Laminar shear stress (LSS) due to pulsatile blood flow enhances endothelial NO production. LSS activates NOS3 directly through a calcium-dependent calmodulin binding and phosphorylation of NOS3 on multiple sites including $\operatorname{Ser}^{1177}$ [5]. Recent studies further demonstrated that chronic LSS increases the expression of not only NOS3 but also argininosuccinate synthetase 1 (ASS1) involved in the provision of L-arginine substrate to NOS3 [6]. ASS1 and NOS3 are co-localized at subcellular compartments in endothelial cells, constituting "citrulline-NO cycle" which also includes argininosuccinate lyase (ASL), and cationic amino acid transporter 1 (CAT1) [7]. It is believed that certain strategies that enhance the endothelial citrulline-NO cycle may be potentially useful to enhance endothelial function.

The so-called French paradox describes the unpredictably low incidence of myocardial infarction in the French despite having a diet rich of calories and fat [8]. Many different hypotheses have been proposed to explain this discrepancy and the most famous one is the theory based on the higher consumption of wine in 
France than in other comparable countries [9]. The vascular protective effects of red wine could be attributed to the compounds derived from grapes because de-alcoholized red wine showed similar effects [10]. Resveratrol is one of the phenolic compounds contained in red wine, and has been postulated as the active compound that provides vascular protective effects $[11,12]$.

Recent studies have established Kruppel-like factor (KLF) 2 and/or KLF4 as directors of the endothelial gene expression program in response to LSS, thus contributing to the establishment of vasodilatory, anti-inflammatory and anti-thrombotic microenvironments $[13,14]$. Indeed, KLF2 and KLF4 appear to be the key upstream regulators of NOS3 and ASS1 gene expressions in response to LSS [15]. Of interest, resveratrol has been observed to stimulate KLF2 and/or KLF4 [16,17]. The aim of the present study was to directly compare the effects of these mechanical (LSS) and pharmacological (resveratrol) stimuli on the citrulline-NO cycle in endothelial cells.

\section{MATERIALS AND METHODS}

\subsection{Cell Culture and Treatments}

Human umbilical vein endothelial cells (HUVECs) were obtained from Clonetics Cambrex (Rockland, ME, USA) and were cultured as previously described [15]. For mechanical LSS treatment, HUVECs, cultured to confluency in $100 \mathrm{~mm}$-culture dishes, were exposed to steady LSS at $12 \mathrm{dyn} \cdot \mathrm{cm}^{-2}$ for $24 \mathrm{~h}$ in a growth medium. LSS was provided by rotating a Teflon cone $\left(0.5^{\circ}\right.$ cone angle $)$ mounted onto a culture dish, as previously described [18]. Control cells were kept under static conditions. For pharmacological treatments, resveratrol (Sigma-Aldrich, St. Louis, MO, USA) and mevastatin (Santa Cruz Biotech, Santa Cruz, CA, USA) were each dissolved in dimethylsulfoxide and added to the culture media at the desired concentrations. Control cells were treated with an equivalent volume of vehicle, and the final concentration of vehicle solvent did not exceed $0.1 \%(\mathrm{v} / \mathrm{v})$. Cell viability was assayed using 3-[4,5-dimethylthiazol-2-yl]-2,5-diphenyltetrazolium bromide (MTT) [19].

\subsection{Real Time Polymerase Chain Reaction (RT-PCR) Analysis}

Total cellular RNA was extracted from the control and treated cells using the RNeasy kit (Qiagen, Valencia, CA, USA). To prepare cDNA, one $\mu \mathrm{g}$ of cellular mRNA was reverse-transcribed using the High Capacity cDNA Archive Kit (Applied Biosystems, Foster city, CA, USA) in accordance with the manufacturer's instructions. The kit utilizes random hexamers primers and MultiScribe ${ }^{\mathrm{TM}}$ Reverse Transcriptase. PCR was conducted using the
StepOnePlus ${ }^{\mathrm{TM}}$ Real-Time PCR System (Applied Biosystems) in a reaction mixture $(20 \mu \mathrm{l})$ containing SYBR $\mathbb{R}$ Green PCR Master Mix (Applied Biosystems), 60 ng cDNA, and 2 picomole of gene-specific primer sets (Macrogen, Seoul, Korea). The reactions were performed with the following conditions: $50^{\circ} \mathrm{C}$ for $2 \mathrm{~min}, 95^{\circ} \mathrm{C}$ for $10 \mathrm{~min}, 40$ cycles of $95^{\circ} \mathrm{C}$ for $15 \mathrm{~s}$ and $60^{\circ} \mathrm{C}$ for $1 \mathrm{~min}$, followed by a dissociation protocol. Single peaks in the melting curve analysis supported the homogeneity of the amplicons. The mRNA expression level relative to the internal control glyceraldehyde 3-phosphate dehydrogenase (GAPDH) was calculated by the comparative threshold cycle $\left(C_{T}\right)$ method [20]. The sequences of the PCR primers were: ASS1 (GeneBank accession number, NM_000050.4) 5'-TTG GAA TGA AGT CCC GAG GTA-3' (sense) and 5'-CTC AGG GCT GTG CCA GAA AC-3' (antisense); ASL(NM_000048.3) 5'-TGA TGC CCC AGA AGA AAA ACC-3'(sense) and 5'-GCA CGG CAC TCA TAG TGT CTG A-3'(antisense); NOS3 (NM 000603.3) 5'-CAG CCT CAC TCC TGT TTT CCA-3' (sense) and 5'-TCA CTC GCT TCG CCA TCA C-3' (antisense); CAT1 (NM_003045.4) 5'-CGC TGC CTG AAC ACT TTT GA-3' (sense) and 5'-AGC ACC AAA CTC GCC ATA GC-3' (antisense); KLF2 (NM

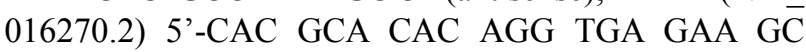
-3'(sense) and 5'-GCA CAG ATG GCA CTG GAA TG -3'(antisense); KLF4 (NM_004235) 5'-CTG CGG CAA AAC CTA CAC AA-3'(sense) and 5'-CCG TCC CAG TCA CAG TGG TA-3'(antisense); GAPDH (NM 002046.3) 5'-ATG GGG AAG GTG AAG GTC G-3', (sense) and 5'-GGG GTC ATT GAT GGC AAC AAT A3' (antisense).

\subsection{Western Blot Analysis}

Cells were subjected to lysis in a buffer (10 mM Tris-Cl, $150 \mathrm{mM} \mathrm{NaCl}, 5 \mathrm{mM}$ EDTA, $0.1 \%$ sodium dodecyl sulfate, $1 \%$ TritonX-100, and $1 \%$ deoxycholate, $\mathrm{pH} 7.2$ ) supplemented with $1 \mathrm{mM}$ phenylmethylsulfonyl fluoride and protease inhibitor cocktail (Roche, Mannheim, Germany). Western blotting was performed as previously described [15]. Mouse monoclonal antibody for NOS3 was purchased from BD Transduction Laboratories (San Diego, CA, USA). Rabbit polyclonal antibody for CAT1 was purchased from Abcam (Cambridge, MA, USA). Goat polyclonal antibody for ASS1, mouse monoclonal antibody for ASL and rabbit polyclonal antibody for KLF4 were from Santa Cruz Biotech (Santa Cruz, CA, USA). Rabbit polyclonal antibody for Ser ${ }^{177}$-phosphorylated $\mathrm{NOS}_{3}$ were from Cell Signaling (Danvers, MA, USA). Mouse monoclonal $\beta$-actin antibody was purchased from Sigma-Aldrich. Goat anti-mouse and anti rabbit secondary antibodies conjugated to horseradish peroxidase were purchased from Cell signaling and Santa 
Cruz Biotech, respectively.

\subsection{NO Production Assay}

NO production from endothelial cells was determined by measuring the NO metabolites, nitrite plus nitrate. Cells were treated with LSS $\left(12 \mathrm{dyn} \cdot \mathrm{cm}^{-2}\right)$, resveratrol $(50 \mu \mathrm{M})$ or mevastatin $(10 \mu \mathrm{M})$ for $24 \mathrm{~h}$ in a phenol red-free Dulbecco's modified Eagle's medium (DMEM) (SigmaAldrich) containing $0.5 \%$ FBS and $25 \mathrm{mM}$ Hepes $(\mathrm{pH}$ 7.4). The conditioned medium was then harvested and analyzed using an amperometric AmiNO700 sensor (Innovative Instruments Inc., Tampa, FL, USA), as previously described [21]. Current changes due to NO generation were monitored and the values were corrected for the background levels of NO metabolites in the medium itself and normalized to the total protein contents of the cells.

\subsection{Statistical Analysis}

Data are presented as the means \pm SEM for experiments performed in triplicate. Significant differences among the groups were determined using the one-way ANOVA at a significance level of $p<0.05$.

\section{RESULTS}

The endothelial citrulline-NO cycle consists of ASS1, ASL, NOS3 and CAT1, as shown in Figure 1(A). ASS1 catalyzes the ATP-dependent condensation reaction of L-citrulline and aspartic acid, producing L-argininosuccinate. ASL catalyzes the hydrolysis of L-argininosuccinate to L-arginine and succinic acid. The L-arginine formed in this reaction is used by NOS3 which generates L-citrulline and NO. CAT1 is involved in transport of L-arginine from the blood into the cells.

In the first experiment, the effects of LSS on the endothelial citrulline-NO cycle were examined. HUVECs were exposed to LSS at $12 \mathrm{dyn} \cdot \mathrm{cm}^{-2}$ for $24 \mathrm{~h}$ or kept under static conditions for the same period, and cellular mRNAs were subjected to quantitative real time PCR analysis to determine the gene expression levels of ASS1, ASL, NOS3, and CAT1. The data were normalized by comparing their expression levels with that of GAPDH, a
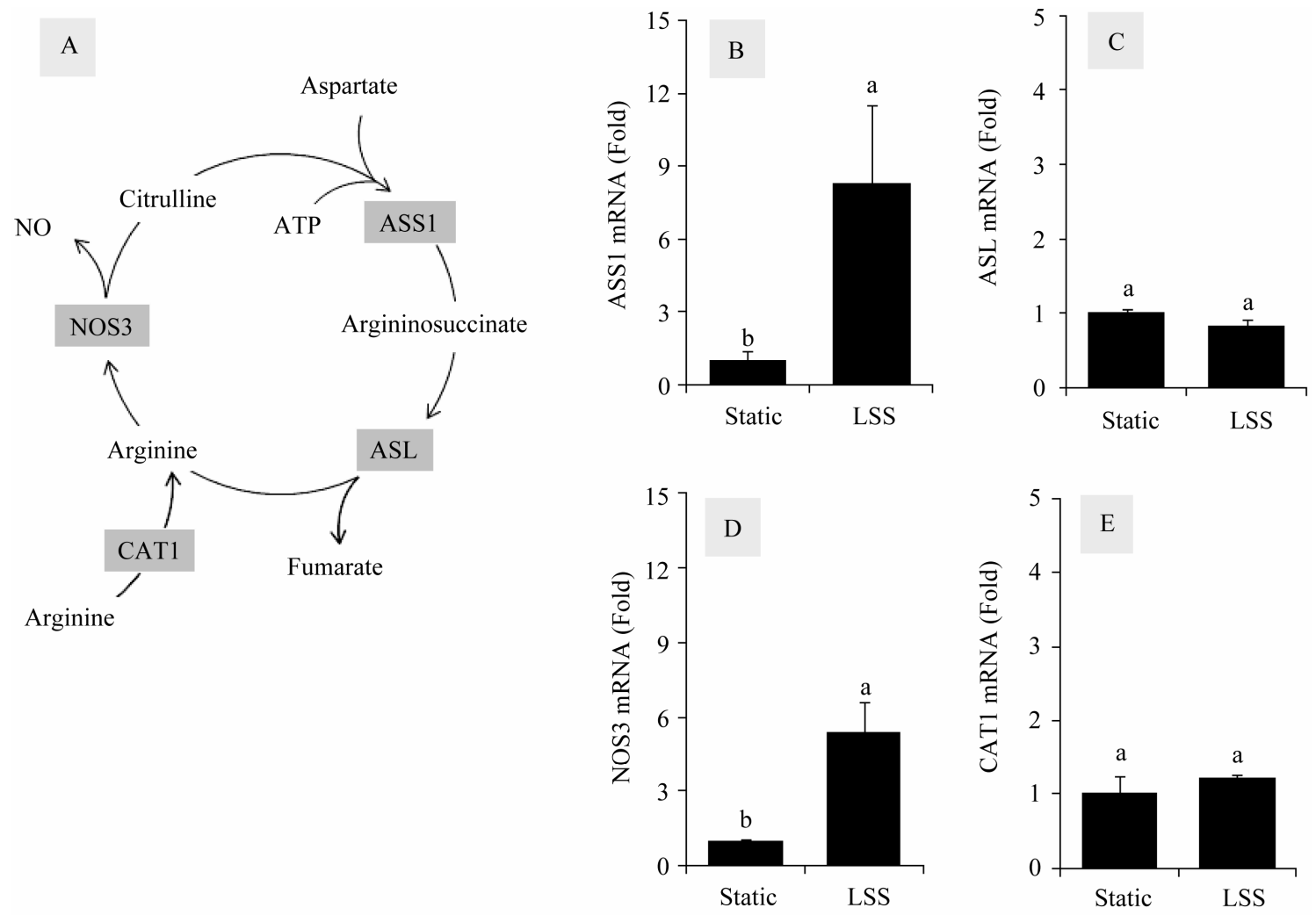

Figure 1. Effects of LSS on the mRNA levels of the citrulline-NO cycle members in HUVECs. The citrulline-NO cycle consists of ASS1, ASL, NOS3 and CAT1 (A). HUVECs were exposed to LSS at $12 \mathrm{dyn} \cdot \mathrm{cm}^{-2}$ for $24 \mathrm{~h}$ or kept under static conditions for the same period, and cellular mRNAs were subjected to quantitative RT-PCR analysis to determine gene expression levels of ASS1 (B), ASL (C), NOS3 (D), and CAT1 (E). The data were normalized by comparing their expression levels with that of GAPDH, a house keeping enzyme. Data are presented as foldchanges compared to static control groups (Means $\pm \mathrm{SEM}, \mathrm{n}=3$ ). Data not sharing the same letters (a or $\mathrm{b}$ on the bars) are statistically different from each other $(p<0.05)$. 
house keeping enzyme. As shown in Figure 1, LSS increased the mRNA levels of ASS1 (8.3 fold) and NOS3 (5.4 fold), but not those of ASL and CAT1. These results confirm previous observations that LSS increases ASS1 and NOS3 expressions [6,22].

In the next experiment, the effects of resveratrol on gene expression of the citrulline-NO cycle members were examined in HUVECs. Cells were treated with resveratrol up to $100 \mu \mathrm{M}$ (cell viability, $80 \%$ ) to determine its effects on the gene expressions. As shown in Figure 2, resveratrol increased the mRNA levels of ASS1 in a dose-dependent manner ( 3.8 fold at $100 \mu \mathrm{M})$ whereas no increases were observed with those of NOS3, ASL and CAT1. The effect of $10 \mu \mathrm{M}$ mevastatin (cell viability, $72 \%$ ) on the mRNA levels of ASS1, ASL, NOS3, and CAT1 were also examined for a comparative purpose. As shown in Figure 2, the stimulatory effects of mevastatin were observed with both ASS1 (2.2-fold increase) and NOS3 (2.9-fold increase). The mRNA levels of ASL and CAT1 were virtually unchanged.

As ASS1 and NOS3 appear to be more responsive than other members of the citrulline-NO cycle to the external stimuli, the effects of LSS, resveratrol and mevastatin on the mRNA levels of KLF2 and KLF4, the upstream regulators of ASS1 and NOS3 expressions, were compared. As shown in Figure 3, the highest fold changes were observed with the LSS treatment that increased KLF2 and KLF4 expressions by 13.2 and 65.1 folds, respectively. Resveratrol $(100 \mu \mathrm{M})$ increased KLF2 and KLF4 expressions by 4.1 and 4.2 folds, respectively. Mevastatin $(10 \mu \mathrm{M})$ increased KLF2 and KLF4 expressions by 5.5 and 19.7 folds, respectively.

Western blot analysis was performed to monitor the protein levels of ASS1, NOS3, ASL, CAT1, KLF4 and $\beta$-actin affected by LSS (12 $\left.\mathrm{dyn} \cdot \mathrm{cm}^{-2}\right)$, resveratrol (50 $\mu \mathrm{M})$ and mevastatin $(10 \mu \mathrm{M})$ (Figure 4). LSS increased the protein levels of ASS1 and NOS3 markedly. ASS1 protein levels were also increased moderately by resveratrol and mevastatin. Mevastatin also increased NOS3 protein level. KLF4 protein levels were highly increased by LSS and mevastatin in that order. The phosphorylation of Ser $^{1177}$ of NOS3 was increased by resveratrol as well as LSS.

Finally, the effects of LSS, resveratrol and mevastatin
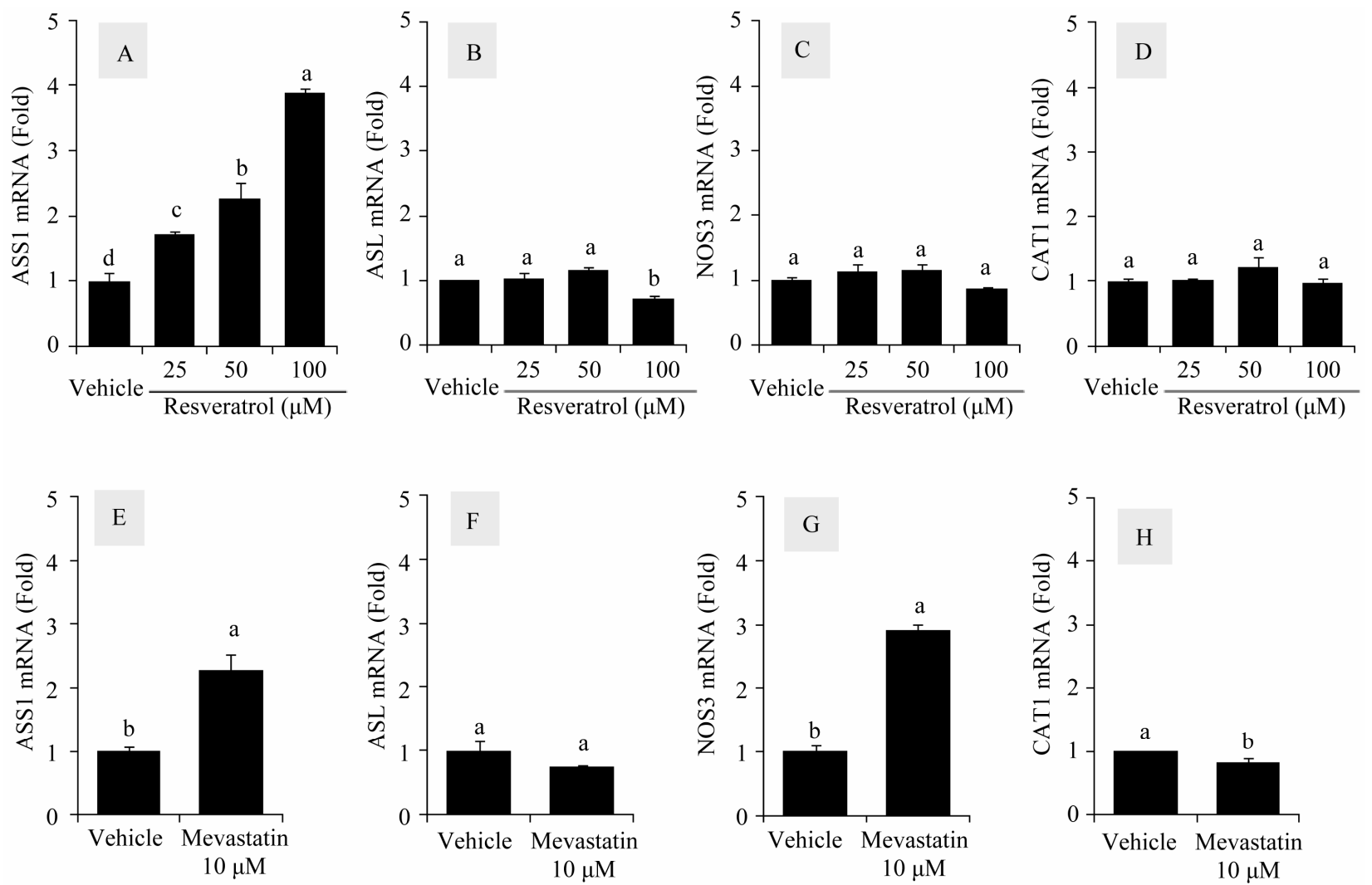

Figure 2. Effects of resveratrol and mevastatin on the mRNA levels of the citrulline-NO cycle members in HUVECs. Cells were treated with resveratrol at 25,50 and $100 \mu \mathrm{M}$ for $24 \mathrm{~h}$ to determine its effects on mRNA expressions of ASS1 (A), ASL (B), NOS3 (C) and CAT1 (D). The effect of mevastatin $(10 \mu \mathrm{M})$ on the mRNA levels of ASS1 (E), ASL (F), NOS3 (G) and CAT1 (H) were examined for comparative purposes. The data were normalized to GAPDH mRNA levels and presented as fold-changes compared to vehicle control groups (Means \pm SEM, $n=3$ ). Data not sharing the same letters $(a, b$, $c$ or $d$ on the bars) are statistically different from each other $(p<0.05)$. 

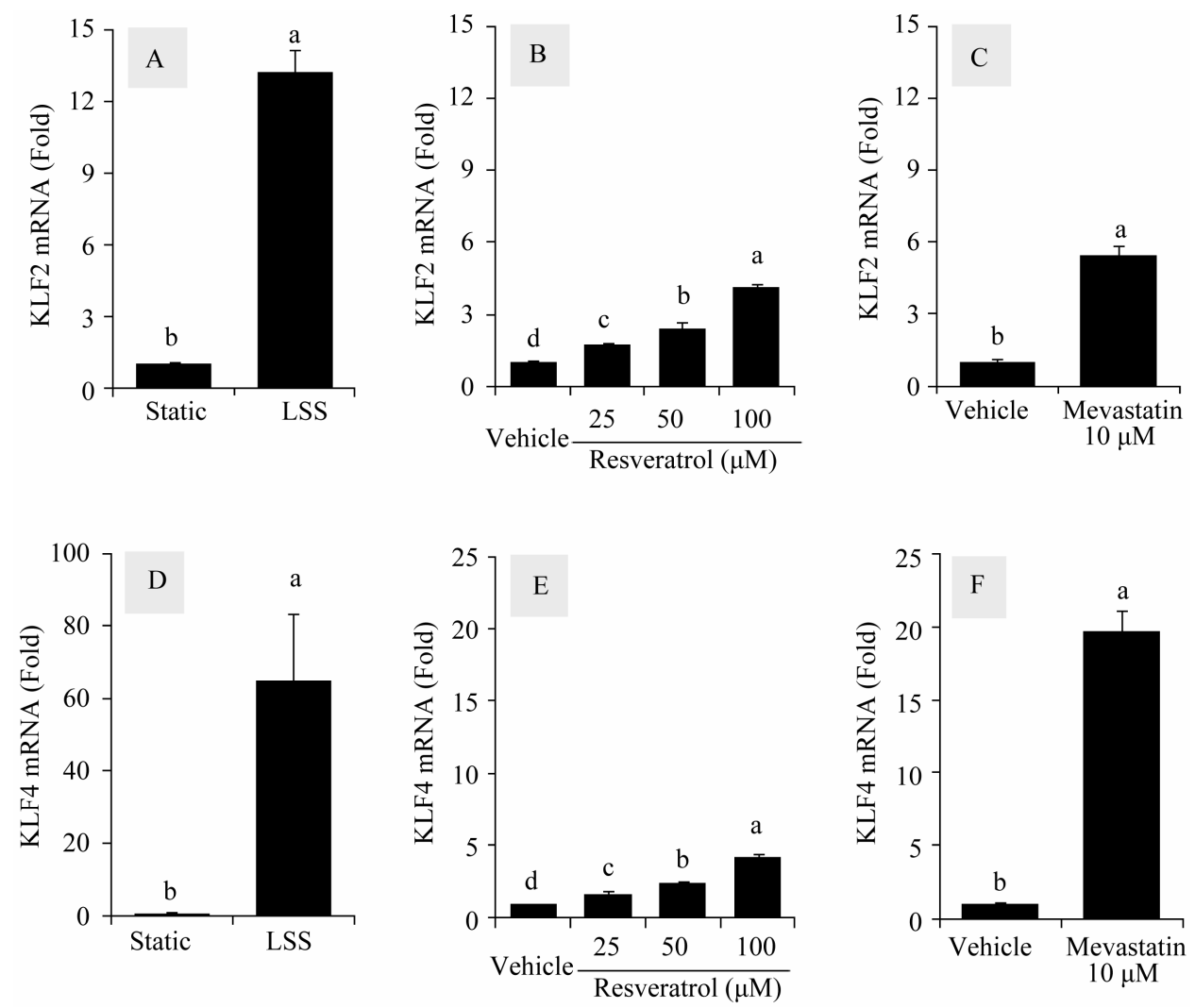

Figure 3. Effects of LSS, resveratrol and mevastatin on the mRNA levels of KLF2 and KLF4 in HUVECs. The effects of LSS, resveratrol and mevastatin on the mRNA levels of KLF2 and KLF4 as upstream regulators of ASS1 and NOS3 were compared. Cells were treated with LSS (12 dyn $\left.\cdot \mathrm{cm}^{-2}\right)(\mathrm{A})(\mathrm{D})$, resveratrol $(25-100 \mu \mathrm{M})(\mathrm{B})(\mathrm{E})$ and mevastatin $(10 \mu \mathrm{M})(\mathrm{C})(\mathrm{F})$ for $24 \mathrm{~h}$ to determine their effects on mRNA expressions of KLF2 (A, B, C) and KLF4 (D) (E) (F). The data were normalized to GAPDH mRNA levels and presented as fold-changes compared to static or vehicle control groups in each experiment (Means \pm SEM, $n=3$ ). Data not sharing the same letters $(\mathrm{a}, \mathrm{b}, \mathrm{c}$ or $\mathrm{d}$ on the bars) are statistically different from each other $(p<0.05)$.

on endothelial NO production in the cells were compared (Figure 5). LSS treatment led to a robust increase of NO production by 6.9 fold compared to the static control cells. Mevastatin also increased NO production by 2.4 fold but resveratrol did not. Effect of resveratrol on NO production was also examined in combination with LSS. Resveratrol did not enhance NO production stimulated by LSS significantly.

\section{DISCUSSION}

NOS3 enzyme uses L-arginine as the sole substrate to produce NO in endothelial cells. Cytosolic concentration of L-arginine in endothelial cells $(>800 \mu \mathrm{M})$ is much higher than NOS3's Michaelis constant (Km) $(\sim 2.9 \mu \mathrm{M})$, but NOS3 activity does not appear to be saturated normally [23]. To explain this observation, it has been suggested that the subcellular local concentration of L-arginine directly available to the NOS3 enzyme may be lower than the average value of the entire cell [24]. As a rate limiting enzyme of this citrulline-NO cycle, ASS1 can regulate NO production in endothelial cells [25]. Other members of the endothelial citrulline-NO cycle, ASL and CAT1 are also functionally closely associated with endothelial NO production [26,27]. Thus, it is important to understand overall changes of the citrullineNO cycle in response to physiologically relevant external stimuli.

It is of interest to directly compare the effects of mechanical LSS treatments and pharmacological resveratrol treatments on the endothelial citrulline-NO cycle in association with NO production. Indeed, these mutually different mechanical and pharmacological factors appear to share many common properties in the regulation of various genes. Previous studies of this laboratory indicated that LSS stimulated the expression of ASS1 and NOS3 by a KLF4-dependent mechanism [15]. Other studies have reported that resveratrol increased NOS3 expression [28] and it stimulated the KLF2/4 signaling pathway $[16,17]$.

In the present study, LSS appeared to strongly stimu- 

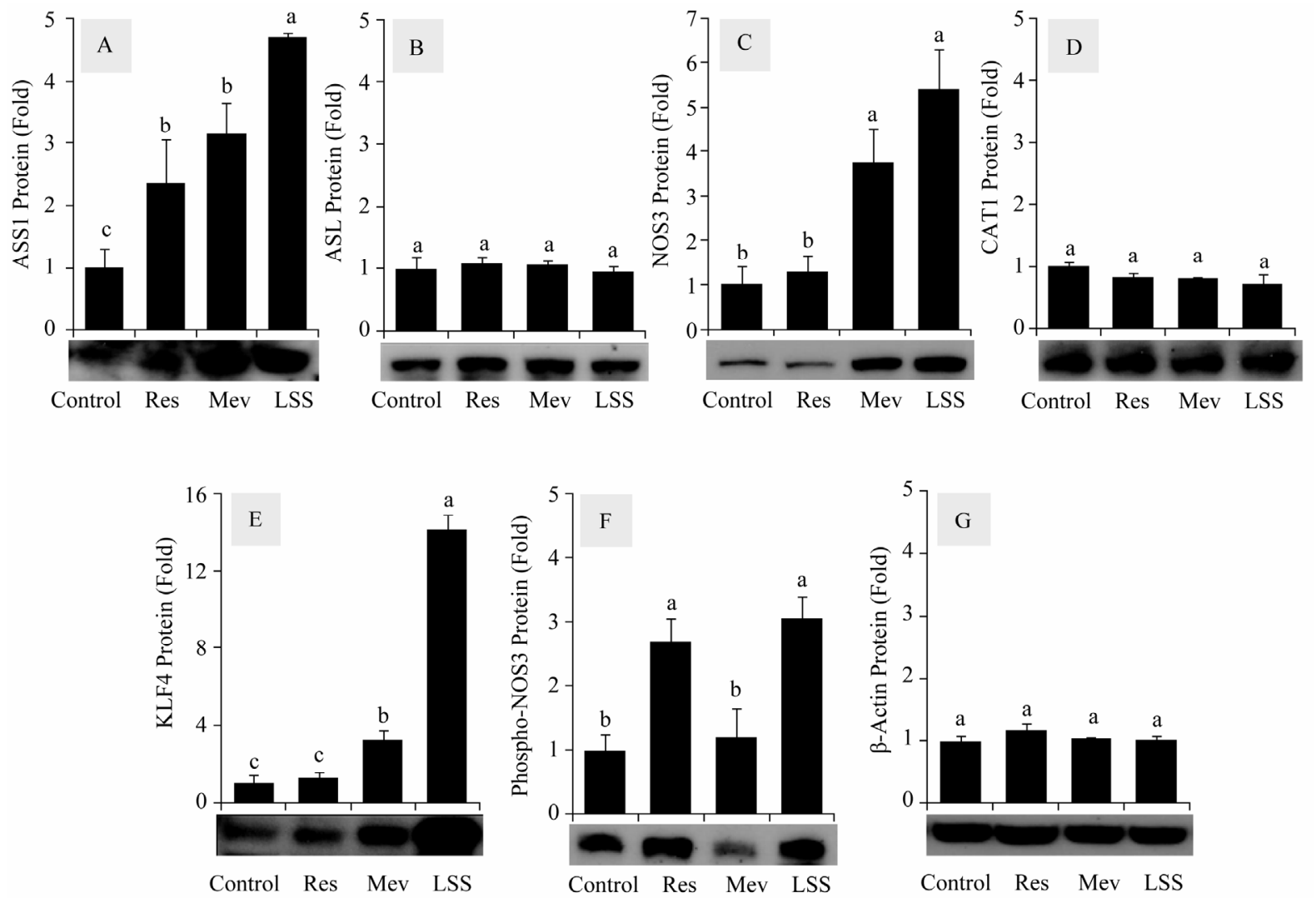

Figure 4. Effects of resveratrol, mevastatin and LSS on the protein levels of the citrulline-NO cycle members in HUVECs. Western blot analysis was performed to monitor the protein expression levels of ASS1 (A); ASL (B); NOS3 (C); CAT1 (D); KLF4 (E); Ser ${ }^{177}$-phosphorylated NOS3 (F) affected by resveratrol $(50 \mu \mathrm{M})$, mevastatin $(10 \mu \mathrm{M})$ and LSS $\left(12 \mathrm{dyn} \cdot \mathrm{cm}^{-2}\right)$. $\beta$-Actin was analyzed as a control $(\mathrm{G})$. Data are presented as fold-changes compared to static vehicle control groups (Means \pm SEM, $\mathrm{n}=3$ ). Data not sharing the same letters ( $\mathrm{a}, \mathrm{b}$ or $\mathrm{c}$ on the bars) are statistically different from each other $(p<0.05)$.
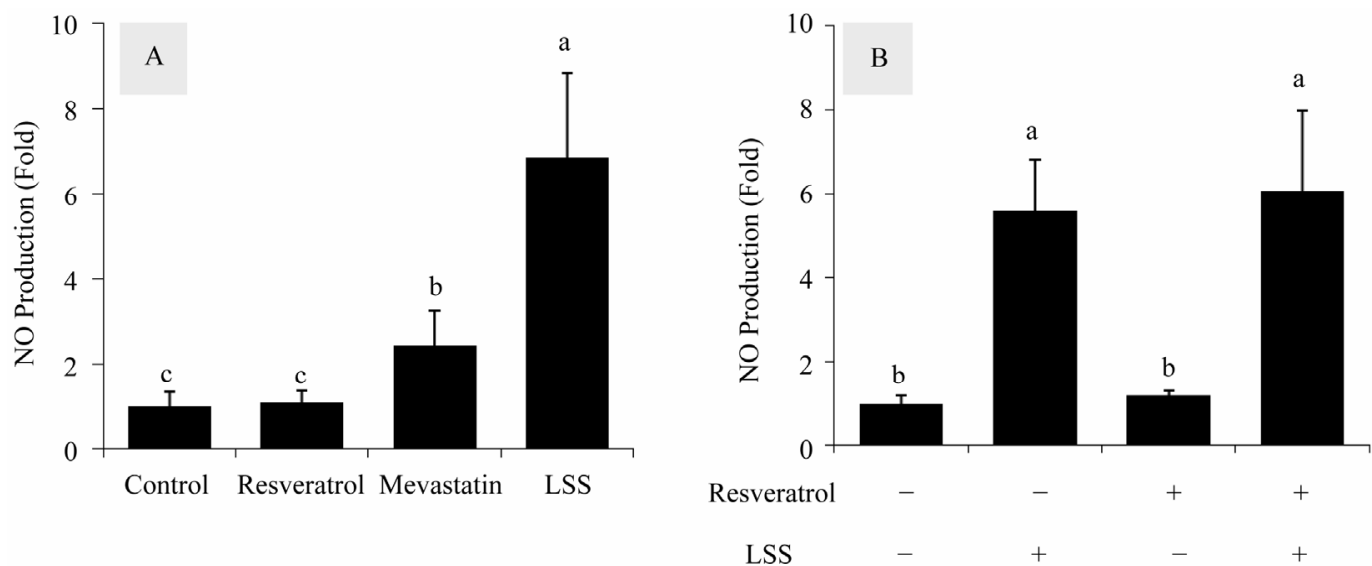

Figure 5. Effects of resveratrol, mevastatin and LSS on NO production in HUVECs. Effects of resveratrol (50 $\mu \mathrm{M})$, mevastatin $(10 \mu \mathrm{M})$ and LSS $\left(12 \mathrm{dyn} \cdot \mathrm{cm}^{-2}\right)$ on NO production were determined (A). Effects of the combination of LSS $\left(12 \mathrm{dyn} \cdot \mathrm{cm}^{-2}\right)$ and resveratrol $(50 \mu \mathrm{M})$ on NO production were also examined (B). Data are presented as fold-changes compared to static vehicle control groups (Means \pm SEM, $n=3-6$ ). Data not sharing the same letters ( $\mathrm{a}, \mathrm{b}$ or $\mathrm{c}$ on the bars) are statistically different from each other $(p<0.05)$.

late the mRNA and protein expression of ASS1 and NOS3 (Figures 1 and 4) whereas resveratrol increased the mRNA and protein expression of ASS1 (Figures 2 and 4). Resveratrol also increased the mRNA expressions of KLF2 and KLF4, the upstream regulators of ASS1 and NOS3 gene expressions, although the changes 
were smaller than those of LSS (Figure 3). Differently from the previous study [28], the stimulatory effects of resveratrol on endothelial NO production were not observed in the present study (Figure 5). Of course, LSS induced a robust NO production. Overall the effects of resveratrol on the endothelial citrulline-NO cycle appeared to be relatively smaller compared to those of LSS. However, because resveratrol was found to enhance ASS1 expression significantly, its pharmacological use might be beneficial when ASS1 activity limits endothelial NO production under certain pathological conditions.

Statins (or HMG-CoA reductase inhibitors) are a class of drugs developed to lower cholesterol synthesis. However, their beneficial effects are not limited to that original purpose. Various statins including mevastatin have been shown to stimulate endothelial NO production by multiple mechanisms [29,30]. Statins are also known to stimulate KLF2 and/or KLF4 [31]. The effects of mevastatin on the endothelial citrulline-NO cycle were observed to be moderate compared to those of LSS and resveratrol (Figures 3 and 4).

The results of the current study showed that LSS had greater effects on the citrulline-NO cycle activity leading to NO production, compared to resveratrol or mevastatin. LSS increased both the protein expression and phosphorylation of NOS3 in addition to ASS1 protein expression. Thus, it is suggested that physical exercise that increases LSS levels [32] might be very important for vascular health. The effects of LSS were partly mimicked by the pharmacological approaches using resveratrol or mevastatin. Both resveratrol and mevastatin increased ASS1 expression. Mevastatin also increased NOS3 protein expression while resveratrol induced NOS3 phosphorylation.

This study highlighted the utility of the endothelial citrulline-NO cycle as a whole as a potential therapeutic target for the enhancement of endothelial function. Among the citrulline-NO cycle members, ASS1 and NOS3 appeared to be sensitively regulated at the transcriptional levels in responsive to mechanical and pharmacological stimuli. Because resveratrol was not so effective as LSS at stimulating the endothelial citrulline-NO cycle, further studies are needed to find more potent drugs that increase the expression of ASS1 and NOS3 genes.

\section{ACKNOWLEDGEMENTS}

This study was supported by a grant of the Korea Health technology R\&D Project, Ministry of Health \& Welfare (Grant No. A111345).

\section{REFERENCES}

[1] Vandenbroucke, E., Mehta, D., Minshall, R. and Malik, A.B. (2008) Regulation of endothelial junctional permeability. Annals of the New York Academy of Sciences,
1123, 134-145. doi:10.1196/annals.1420.016

[2] Vita, J.A. (2011) Endothelial function. Circulation, 124, e906-e912. doi:10.1161/CIRCULATIONAHA.111.078824

[3] Furchgott, R.F. and Zawadzki, J.V. (1980) The obligatory role of endothelial cells in the relaxation of arterial smooth muscle by acetylcholine. Nature, 288, 373-376. doi: $10.1038 / 288373 \mathrm{a} 0$

[4] Bredt, D.S. (1999) Endogenous nitric oxide synthesis: Biological functions and pathophysiology. Free Radical Research, 31, 577-596. doi:10.1080/10715769900301161

[5] Boo, Y.C. and Jo, H. (2003) Flow-dependent regulation of endothelial nitric oxide synthase: Role of protein kinases. American Journal of Physiology-Cell Physiology, 285, C499-C508.

http://www.ncbi.nlm.nih.gov/pubmed/12900384

[6] Mun, G.I., Lee, S.J., An, S.M., Kim, I.K. and Boo, Y.C. (2009) Differential gene expression in young and sensecent endothelial cells under static and laminar shear stress conditions. Free Radical Biology \& Medicine, 47, 291299. doi:10.1016/j.freeradbiomed.2009.04.032

[7] Flam, B.R., Eichler, D.C. and Solomonson, L.P. (2007) Endothelial nitric oxide production is tightly coupled to the citrulline-NO cycle. Nitric Oxide, 17, 115-121. doi:10.1016/j.niox.2007.07.001

[8] Simini, B. (2000) Serge renaud: From French paradox to Cretan miracle. Lancet, 355, 48. doi:10.1016/S0140-6736(05)71990-5

[9] Opie, L.H. and Lecour, S. (2007) The red wine hypothesis: From concepts to protective signalling molecules. Euro- pean Heart Journal, 28, 1683-1693. doi:10.1093/eurheartj/ehm149

[10] Wang, Z., Zou, J., Cao, K., Hsieh, T.C., Huang, Y. and Wu, J.M. (2005) Dealcoholized red wine containing known amounts of resveratrol suppresses atherosclerosis in hypercholesterolemic rabbits without affecting plasma lpid levels. International Journal of Molecular Medicine, 16, 533-540. http://www.ncbi.nlm.nih.gov/pubmed/16142383

[11] Petrovski, G., Gurusamy, N. and Das, D.K. (2011) Resveratrol in cardiovascular health and disease. Annals of the New York Academy of Sciences, 1215, 22-33. doi:10.1111/j.1749-6632.2010.05843.x

[12] Wu, J.M. and Hsieh, T.C. (2011) Resveratrol: A cardioprotective substance. Annals of the New York Academy of Sciences, 1215, 16-21. doi:10.1111/j.1749-6632.2010.05854.x

[13] Dekker, R.J., van Soest, S., Fontijn, R.D., Salamanca, S., de Groot, P.G., VanBavel, E., Pannekoek, H. and Horrevoets, A.J. (2002) Prolonged fluid shear stress induces a distinct set of endothelial cell genes, most specifically lung Kruppel-like factor (KLF2). Blood, 100, 1689-1698. doi:10.1182/blood-2002-01-0046

[14] Parmar, K.M., Larman, H.B., Dai, G., Zhang, Y., Wang, E.T., Moorthy, S.N., Kratz, J.R., Lin, Z., Jain, M.K., Gimbrone Jr., M.A. and Garcia-Cardena, G. (2006) Integration of flow-dependent endothelial phenotypes by Kruppel-like factor 2. Journal of Clinical Investigation, 
116, 49-58. doi:10.1172/JCI24787

[15] Mun, G.I. and Boo, Y.C. (2012) A regulatory role of Kruppel-like factor 4 in endothelial argininosuccinate synthetase 1 expression in response to laminar shear stress. Biochemical and Biophysical Research Communications, 420, 450-455. doi:10.1016/j.bbrc.2012.03.016

[16] Villarreal Jr., G., Zhang, Y., Larman, H.B., Gracia-Sancho, J., Koo, A. and Garcia-Cardena, G. (2010) Defining the regulation of KLF4 expression and its downstream transcriptional targets in vascular endothelial cells. Biochemical and Biophysical Research Communications, 391, 984-989. doi:10.1016/j.bbrc.2009.12.002

[17] Gracia-Sancho, J., Villarreal Jr., G., Zhang, Y. and Garcia-Cardena, G. (2010) Activation of SIRT1 by resveratrol induces KLF2 expression conferring an endothelial vasoprotective phenotype. Cardiovascular Research, 85, 514-519. doi:10.1093/cvr/cvp337

[18] Boo, Y.C., Sorescu, G., Boyd, N., Shiojima, I., Walsh, K., Du, J. and Jo, H. (2002) Shear stress stimulates phosphorylation of endothelial nitric-oxide synthase at Ser1179 by Akt-independent mechanisms: Role of protein kinase A. Journal of Biological Chemistr, 277, 33883396. doi:10.1074/jbc.M108789200

[19] Denizot, F. and Lang, R. (1986) Rapid colorimetric assay for cell growth and survival. Modifications to the tetrazolium dye procedure giving improved sensitivity and reliability. Journal of Immunological Methods, 89, 271-277. doi:10.1016/0022-1759(86)90368-6

[20] Liu, W. and Saint, D.A. (2002) A new quantitative method of real time reverse transcription polymerase chain reaction assay based on simulation of polymerase chain reaction kinetics. Analytical Biochemistry, 302, 5259. doi:10.1006/abio.2001.5530

[21] Boo, Y.C., Tressel, S.L. and Jo, H. (2007) An improved method to measure nitrate/nitrite with an NO-selective electrochemical sensor. Nitric Oxide, 16, 306-312. doi:10.1016/j.niox.2006.09.004

[22] Mun, G.I., An, S.M., Park, H., Jo, H. and Boo, Y.C. (2008) Laminar shear stress inhibits lipid peroxidation induced by high glucose plus arachidonic acid in endothelial cells. American Journal of Physiology-Heart and Circulatory Physiology, 295, H1966-H1973. doi:10.1152/ajpheart.00727.2008

[23] Cooke, J.P. and Tsao, P.S. (1997) Arginine: A new therapy for atherosclerosis? Circulation, 95, 311-312. doi:10.1161/01.CIR.95.2.311

[24] McDonald, K.K., Zharikov, S., Block, E.R. and Kilberg, M.S. (1997) A caveolar complex between the cationic amino acid transporter 1 and endothelial nitric-oxide synthase may explain the "arginine paradox". Journal of Biological Chemistry, 272, 31213-31216. doi:10.1074/jbc. 272.50 .31213

[25] Goodwin, B.L., Solomonson, L.P. and Eichler, D.C. (2004) Argininosuccinate synthase expression is required to maintain nitric oxide production and cell viability in aortic endothelial cells. Journal of Biological Chemistry, 279, 18353-18360. doi:10.1074/jbc.M308160200

[26] Erez, A., Nagamani, S.C., Shchelochkov, O.A., Premkumar, M.H., Campeau, P.M., Chen, Y., Garg, H.K., Li, L., Mian, A., Bertin, T.K., Black, J.O., Zeng, H., Tang, Y., Reddy, A.K., Summar, M., O'Brien, W.E., Harrison, D.G., Mitch, W.E., Marini, J.C., Aschner, J.L., Bryan, N.S. and Lee, B. (2011) Requirement of argininosuccinate lyase for systemic nitric oxide production. Nature Medicine, 17, 1619-1626. doi:10.1038/nm.2544

[27] Li, C., Huang, W., Harris, M.B., Goolsby, J.M. and Venema, R.C. (2005) Interaction of the endothelial nitric oxide synthase with the CAT-1 arginine transporter enhances NO release by a mechanism not involving arginine transport. Biochemical Journal, 386, 567-574. doi:10.1042/BJ20041005

[28] Wallerath, T., Deckert, G., Ternes, T., Anderson, H., Li, H., Witte, K. and Forstermann, U. (2002) Resveratrol, a polyphenolic phytoalexin present in red wine, enhances expression and activity of endothelial nitric oxide synthase. Circulation, 106, 1652-1658. doi:10.1161/01.CIR.0000029925.18593.5C

[29] Amin-Hanjani, S., Stagliano, N.E., Yamada, M., Huang, P.L., Liao, J.K. and Moskowitz, M.A. (2001) Mevastatin, an HMG-CoA reductase inhibitor, reduces stroke damage and upregulates endothelial nitric oxide synthase in mice. Stroke, 32, 980-986. doi:10.1161/01.STR.32.4.980

[30] Hernandez-Perera, O., Perez-Sala, D., Navarro-Antolin, J., Sanchez-Pascuala, R., Hernandez, G., Diaz, C. and Lamas, S. (1998) Effects of the 3-hydroxy-3-methylglutaryl-CoA reductase inhibitors, atorvastatin and simvastatin, on the expression of endothelin-1 and endothelial nitric oxide synthase in vascular endothelial cells. Journal of Clinical Investigation, 101, 2711-2719. doi:10.1172/JCI1500

[31] Sen-Banerjee, S., Mir, S., Lin, Z., Hamik, A., Atkins, G.B., Das, H., Banerjee, P., Kumar, A. and Jain, M.K. (2005) Kruppel-like factor 2 as a novel mediator of statin effects in endothelial cells. Circulation, 112, 720-726. doi:10.1161/CIRCULATIONAHA.104.525774

[32] Padilla, J., Harris, R.A., Rink, L.D. and Wallace, J.P. (2008) Characterization of the brachial artery shear stress following walking exercise. Vascular Medicine, 13, 105111. doi: $10.1177 / 1358863 \times 07086671$ 